\title{
Structured nursing educational programs improve quality of life outcomes in patients with Cushing's syndrome
}

\author{
Sofia Llahana ${ }^{1,2}$ (1) $\cdot$ Nicola Thomas ${ }^{3}$
}

Received: 16 December 2015/Accepted: 18 March 2016/Published online: 5 April 2016

(C) Springer Science+Business Media New York 2016

Impaired quality of life (QoL) has been reported in patients with Cushing's syndrome even after biochemical cure, with worse QoL outcomes compared to patients with other pituitary adenomas [1]. A variety of physical, neuropsychosomatic, psychological, and sociodemographic factors have been found to influence QoL parameters in patients with Cushing's syndrome [1, 2]. A systematic review by Andela et al. found that QoL outcomes such as physical functioning, bodily pain, and general health improved after surgical or pharmaceutical interventions in 6 of the 8 studies involving patients with Cushing's syndrome [1].

Despite the well-documented impaired QoL in patients with Cushing's syndrome, the prospective randomized study by Martinez-Momblan et al. [3], discussed in this issue, is the first to provide evidence of the positive effect of an educational intervention program on patients' $\mathrm{QoL}$ outcomes, physical activity, healthy lifestyle habits, sleep patterns, and reduction in pain levels. Moreover, patients with Cushing's syndrome in this study maintained improvements in QoL at 9 months post educational intervention [3].

Nursing educational programs which involved patients with Cushing's syndrome have been documented since the early 1960s but focused mainly on the management of

Sofia Llahana

sofia.1lahana@uclh.nhs.uk

1 Department of Diabetes \& Endocrinology, University College London Hospitals (UCLH) NHS Foundation Trust, 3rd Floor Central Wing, 250 Euston Road, London NW1 2PG, UK

2 Centre for Behavioral Medicine, UCL, London, UK

3 School of Health and Social Care, London South Bank University, London, UK glucocorticoid treatment and prevention of adrenal crisis. Kathleen Shea and colleagues developed a structured educational program which also included a teaching guide for nurses to "...prepare the patient to live intelligently with his regimen of hormonal replacement therapy in both normal and emergency situations" (p. 82) [4].

A more recent study found that a nursing educational program delivered over a 3-h group meeting $(N=246$; $71 \%$ secondary adrenal insufficiency) showed significant improvement $(p<0.05)$ at 6 months post intervention in patients' self-management skills to prevent adrenal crisis [5].

Improved self-management skills for glucocorticoid replacement form a cornerstone in the care of patients with Cushing's syndrome who develop adrenal insufficiency and the above studies emphasize the importance of patient education $[4,5]$. However, these interventions have a narrow focus, and unlike the Martinez-Momblan et al.'s study [3], they do not take into consideration the patient's holistic self-management needs and QoL parameters.

Despite advantages of group educational programs and evidence from Martinez-Momblan et al. [3] in improving QoL, one should not assume that this approach is suitable for all patients with Cushing's syndrome.

Geographical dispersion, time/date restrictions when educational programs are delivered, literacy levels, and patients' comfort levels of learning and interacting within a group may often influence non-attendance. Response rates of $49 \%(N=61)$ [3] and $61 \%(N=246)$ [5] were achieved and it would have been useful for these studies to have explored why patients declined participation in the educational program.

We know that patients' ability to retain information following educational interventions diminishes over time [6], and it is therefore crucial to provide "real time" access 
to information and continuous reinforcement of existing knowledge.

Moreover, patients' readiness and motivation can influence the outcome of educational programs on improving QoL parameters. A strength in Martinez-Momblan et al.'s study regarding QoL outcomes was that patients with Cushing's syndrome entered the program with strong motivation to complete all education sessions and to improve their health [3], although one may also view this as limitation as it can skew the results.

Martinez-Momblan et al. found lower number of unscheduled visits and acute hospital admissions for patients in the intervention group compared to patients in the control group. More importantly, patients in the intervention group reported reduction in unscheduled visit and acute hospital admissions post educational program compared to baseline [3]. This has important implications in justifying health resources required to deliver intense educational programs which can lead to improved QoL outcomes for our patients and lower cost for health services in the long run.

A lack of reliable biochemical parameters to assess changes in QoL outcomes for patients with Cushing's syndrome in remission and with hypopituitarism is a limitation compared to other long-term conditions. This is reflected in a study involving 102 patients with treated Cushing's syndrome where $92 \%$ were biochemically cured but only $80.4 \%$ felt they had achieved remission and continued to report impaired QoL [2].

However, there are validated questionnaires such as the CushingQoL [7] that can detect improvement or deterioration in QoL, and it is crucial that these are adopted in clinical practice to evaluate outcomes of any educational programs.

Martinez-Momblan et al. [3] provide the first piece in the puzzle of research evidence supporting the need for nurse-led multidisciplinary educational programs in Cushing's syndrome. However, there is urgency for further research to develop effective intervention programs which can improve QoL outcomes for patients with Cushing's syndrome and other pituitary conditions.

Educational interventions often lack theoretical underpinning which makes it difficult to generalize findings and adopt their study design to other settings. A recent systematic review on effective teaching strategies for patients with a variety of conditions found that there were challenges in evaluating the success of specific methods of delivery: details of the educational intervention are not fully reported and more importantly outcome measures are not clearly articulated [8].

It is therefore important to adopt a clear theoretical framework which can guide robust research methodology and explain the likely influences of educational interventions on QoL outcomes. These programs should not only aim to improve patient's knowledge and self-management skills but should also adopt psychosocial approaches to address patient motivation, beliefs, and illness perceptions [9].

Tiemensma et al. found that patients with Cushing's syndrome in remission report more negative illness perceptions compared to patients with other chronic conditions and these were strongly related to impaired QoL [10].

As Friedman et al's systematic review suggested, specific individualized education should be provided to patients rather than general information about their condition [8]. Education should be structured (ad-hoc random question and answer sessions are not effective), sessions should involve a variety of teaching strategies, and education for minority groups should be culturally sensitive [8]. We need to be creative and eHealth educational programs may present many opportunities to provide patients with "real time" structured education to complement face-toface care away from the traditional hospital setting.

\section{Compliance with ethical standards}

Conflict of interest S. Llahana has received unrestricted educational Grants from Sandoz UK and lecture/consultancy fees from Shire and MerckSerono. N. Thomas has no conflict of interest.

\section{References}

1. C.D. Andela, M. Scharloo, A.M. Pereira, A.A. Kaptein, N.R. Biermasz, Quality of life (QoL) impairments in patients with a pituitary adenoma: a systematic review of QoL studies. Pituitary 18(5), 752-776 (2015)

2. A. Carluccio, N.K. Sundaram, S. Chablani, L.G. Amrock, J.K. Lambert, K.D. Post, E.B. Geer, Predictors of quality of life in 102 patients with treated Cushing's disease. Clin. Endocrinol. 82, 404-411 (2015)

3. M.A. Martinez-Momblan, C. Gomez, A. Santos, N. Porta, J. Esteve, I. Ubeda, I. Halperin, B. Campillo, M. Guillaumet, S.M. Webb, E. Resmini, A specific nursing educational program in patients with Cushing's syndrome. Endocrine (2015). doi:10. 1007/s12020-015-0737-0

4. K.M. Shea, C.P. O'Connor, E.G. Karafelis, G.W. Thorn, G.P. Kozak, Teaching a patient to live with adrenal insufficiency. Am. J. Nurs. 65(12), 80-85 (1965)

5. H.J.W.J. Repping-Wuts, N.M.M.L. Stikkelbroeck, A. Noordzij, M. Kerstens, A.R.M.M. Hermus, A glucocorticoid education group meeting: an effective strategy for improving self-management to prevent adrenal crisis. Eur. J. Endocrinol. 169(1), 17-22 (2013)

6. I.A. Harsch, A. Schuller, E.G. Hahn, J. Hensen, Cortisone replacement therapy in endocrine disorders: quality of self-care. J. Eval. Clin. Pract. 16(3), 492-498 (2010)

7. S.M. Webb, X. Badia, M.J. Barahona, A. Colao, C.J. Strasburger, A. Tabarin, M.O. van Aken, R. Pivonello, G. Stalla, S.W. Lamberts, J.E. Glusman, Evaluation of health-related quality of life in patients with Cushing's syndrome with a new questionnaire. Eur. J. Endocrinol. 158(5), 623-630 (2008) 
8. A.J. Friedman, R. Cosby, S. Boyko, J. Hatton-Bauer, G. Turnbull, Effective teaching strategies and methods of delivery for patient education: a systematic review and practice guideline recommendations. J. Cancer Educ. 26(1), 12-21 (2011)

9. R. Horne, J. Weinman, N. Barber, R. Elliot, M. Morgan, Concordance, adherence and compliance in medicine taking: a conceptual map and research priorities. National Institute for Health Research (NIHR) Service Delivery and Organisation (SDO)
Programme, London (2005), http://www.nets.nihr.ac.uk/_data/ assets/pdf_file/0007/81394/ES-08-1412-076.pdf. Accessed 29 Jan 2016

10. J. Tiemensma, A.A. Kaptein, A.M. Pereira, J.W. Smit, J.A. Romijn, N.R. Biermasz, Negative illness perceptions are associated with impaired quality of life in patients after long-term remission of Cushing's syndrome. Eur. J. Endocrinol. 165(4), 527-535 (2011) 